\title{
L-citrulline production by metabolically engineered Corynebacterium glutamicum from glucose and alternative carbon sources
}

\author{
Dorit Eberhardt, Jaide V K Jensen and Volker F Wendisch ${ }^{*}$
}

\begin{abstract}
L-citrulline plays an important role in human health and nutrition and is an intermediate of the L-arginine biosynthetic pathway. L-citrulline is a by-product of L-arginine production by Corynebacterium glutamicum. In this study, C. glutamicum was engineered for overproduction of L-citrulline as major product without L-arginine being produced as by-product. To this end, L-arginine biosynthesis was derepressed by deletion of the arginine repressor gene $\arg R$ and conversion of $L$-citrulline towards $L$-arginine was avoided by deletion of the argininosuccinate synthetase gene $\arg G$. Moreover, to facilitate L-citrulline production the gene encoding a feedback resistant $\mathrm{N}$-acetyl L-glutamate kinase $\arg ^{\mathrm{fbr}}$ as well as the gene encoding L-ornithine carbamoylphosphate transferase $\arg F$ were overexpressed. The resulting strain accumulated $44.1 \pm 0.5 \mathrm{mM} \mathrm{L}$-citrulline from glucose minimal medium with a yield of $0.38 \pm 0.01 \mathrm{~g} \cdot \mathrm{g}^{-1}$ and a volumetric productivity of $0.32 \pm 0.01 \mathrm{~g} \cdot \mathrm{l}^{-1} \cdot \mathrm{h}^{-1}$. In addition, production of L-citrulline from the alternative carbon sources starch, xylose, and glucosamine could be demonstrated.
\end{abstract}

Keywords: Corynebacterium glutamicum; L-citrulline; L-arginine; Alternative carbon sources; Starch; Xylose; Glucosamine; Metabolic engineering

\section{Introduction}

L-citrulline is a natural non-proteinogenic amino acid whose name is derived from watermelon Citrullus lanatus (Wada 1930). In mammalians it serves as a precursor for L-arginine. In contrast to the proteinogenic L-arginine, which is not transferred to the blood stream, when ingested, L-citrulline can be converted to $\mathrm{L}$-arginine, which is then released by the kidney into the blood stream. It is applied in several medical approaches e.g. as a pharmaconutrient (Rimando and Perkins-Veazie 2005; Curis et al. 2005).

Currently, biocatalytic and fermentative methods to produce L-citrulline using Pseudomonas putida (Kakimoto et al. 1971; Yamamoto et al. 1974) or Bacillus subtilis strains exist (Okumura et al. 1966). Additionally, extraction processes from watermelon have been established (Fish 2012). L-citrulline is an intermediate of L-arginine biosynthesis and accumulates as a by-product of engineered

\footnotetext{
* Correspondence: volker.wendisch@uni-bielefeld.de Genetics of Prokaryotes, Faculty of Biology \& Center for Biotechnology, Bielefeld University, Universitätsstraße 25, 33615 Bielefeld, Germany
}

L-arginine producing Corynebacterium glutamicum strains (Ikeda et al. 2009; Schneider et al. 2011).

C. glutamicum is a workhorse for amino acid production and is employed for the annual production of several million tons of L-glutamate and L-lysine (Wendisch 2014). C. glutamicum has been engineered to produce a wide range of bioproducts, such as diamines, carotenoids, terpenes, proteins (Schneider and Wendisch 2010; Schneider et al. 2012; Heider et al. 2014a, b; Frohwitter et al. 2014; Kikuchi et al. 2009; Teramoto et al. 2011; An et al. 2013) and the L-glutamate family amino acids L-arginine, L-ornithine, and L-proline (Schneider et al. 2011; Ikeda et al. 2009; Georgi et al. 2005; Blombach et al. 2009; Jensen and Wendisch 2013). However, the production of L-citrulline as the only or major product has not been published yet.

Due to its natural ability to produce L-glutamate under several eliciting conditions, C. glutamicum is a suitable producer of L-glutamate-derived products (Sato et al. 2008; Radmacher et al. 2005; Kim et al. 2009, 2010; Delaunay et al. 1999; Wendisch et al. 2014). L-ornithine is a non-proteinogenic glutamate-family amino acid and 


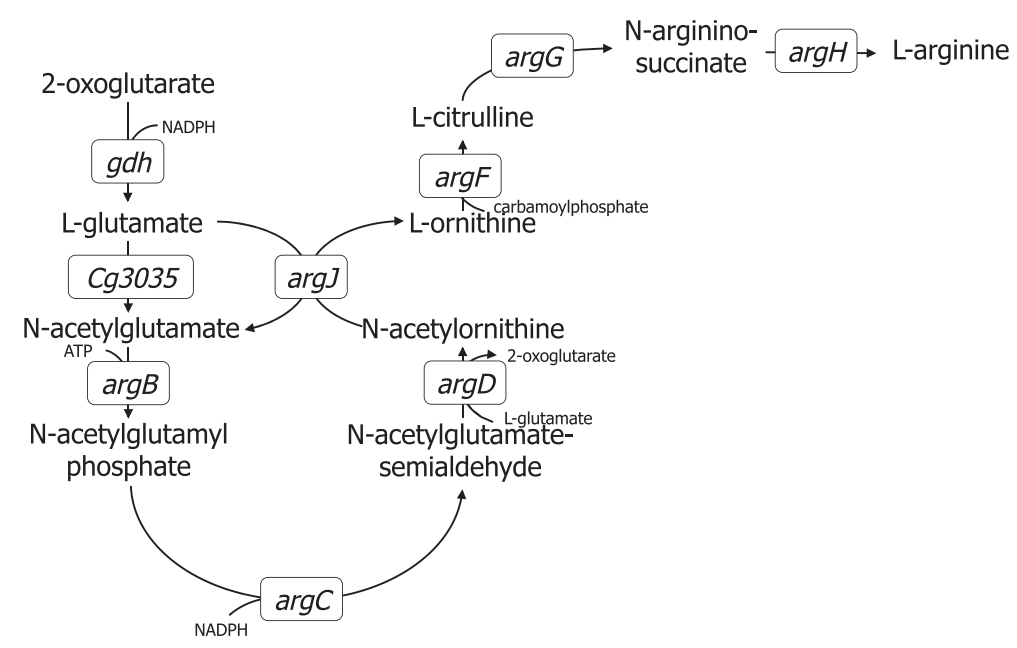

Figure 1 L-arginine pathway in C. glutamicum (modified from (Wendisch et al. 2014)). gdh: L-glutamate dehydrogenase, cg3035: anaplerotic $\mathrm{N}$-acetylL-glutamate synthase, argj: L-ornithine N-acetyltransferase, argB: N-acetylL-glutamate kinase; argC: N-acetyl-gamma-glutamyl-phosphate reductase; $\operatorname{argD}$ : acetylL-ornithine aminotransferase; $\operatorname{argE:}$ acetylL-ornithine deacetylase; argF: L-ornithine carbamoyltransferase; argG: argininosuccinate synthetase; $\operatorname{argH}$ : argininosuccinate lyase. Oxoglutarate is an intermediate of the central carbon metabolism.

an intermediate of L-arginine biosynthesis (Figure 1). An ornithine producer was obtained by deletion of $\arg R$, the gene encoding the genetic repressor of the arginine biosynthesis operon, and $\arg F$ to prevent further processing of ornithine (Schneider et al. 2011). The production of L-proline from L-ornithine is possible by the heterologous overexpression of ocd from Pseudomonas putida, encoding ornithine cyclodeaminase (Jensen and Wendisch 2013). The diamine putrescine can be produced by overexpression of the Escherichia coli gene $s p e C$, which encodes ornithine decarboxylase (Schneider et al. 2012; Schneider and Wendisch 2010). As the arginine biosynthetic pathway is naturally regulated by feedback inhibition of $\mathrm{N}$-acetylglutamate kinase (encoded by $\arg B$ ) by arginine, the use of feedback resistant enzyme variants in combination with deletion of $\arg R$ has been described to overproduce L-arginine (Sakanyan et al. 1996; Ikeda et al. 2009; Schneider et al. 2011).

C. glutamicum can utilize a variety of carbon sources. In contrast to many other microorganisms used in biotechnology, simultaneous utilization of carbon sources e.g. present in mixtures such as lignocellulosic hydrolysates is a hall mark of C. glutamicum (Blombach and Seibold 2010; Meiswinkel et al. 2013a, b). The natural substrate spectrum of C. glutamicum includes monosaccharides, disaccharides, and organic acids as well as alcohols (Blombach and Seibold 2010; Arndt and Eikmanns 2008; Peters-Wendisch et al. 1998; Jolkver et al. 2009; Sasaki et al. 2011). To allow access to alternative carbon sources, C. glutamicum has also been engineered for utilization of glycerol, pentoses, and amino sugars as well as polysaccharides (Schneider et al. 2011; Rittmann et al. 2008; Seibold et al. 2006;
Uhde et al. 2013; Gopinath et al. 2011; Matano et al. 2014).

One aim to reduce production cost is the use of complex sugar substrates for the production of biotechnological products. As an example of using a polymeric raw material without decomposition to its monomeric compounds e.g. by enzyme treatment, soluble starch could be used as a carbon source for the production of L-lysine and organic acids by engineered $C$. glutamicum

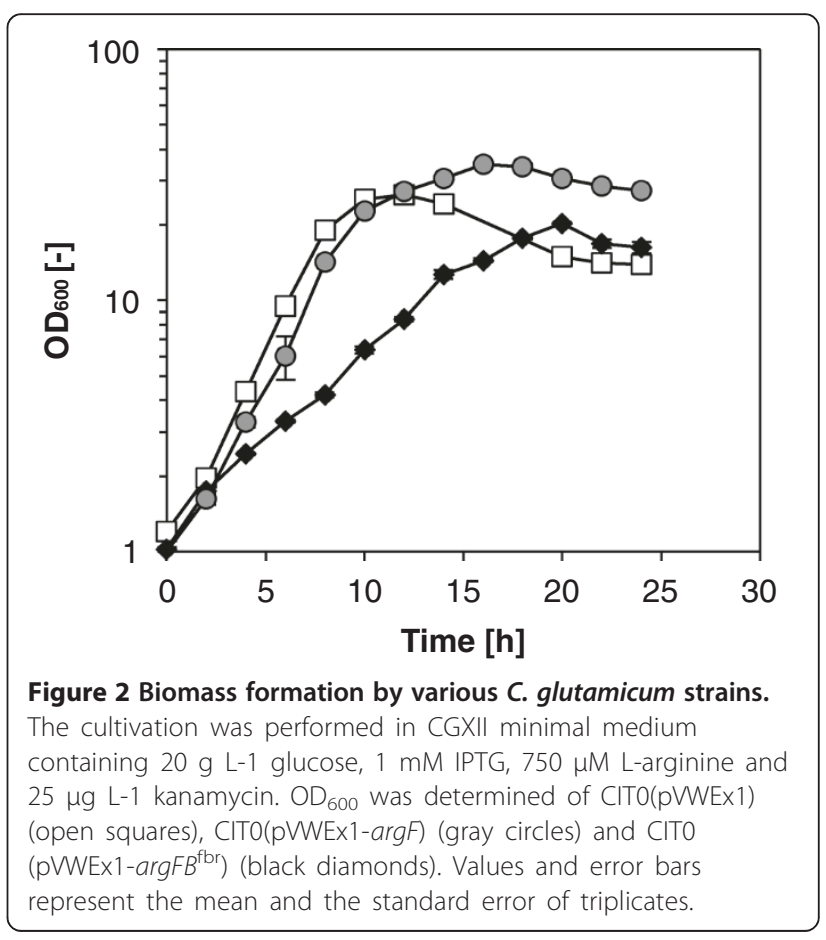


(Seibold et al. 2006; Tateno et al. 2007; Tsuge et al. 2013). However, due to the growing world population and a correlating higher demand for food, biotechnological processes based on non-food derived carbon sources are sought. Xylose is a pentose sugar compound present in the hemicellulosic fraction of agricultural wastes as for example rice straw. Glucosamine, on the other hand, is a constituent of chitin, the second most abundant biopolymer in Nature, which is accessible e.g. from shrimp shell waste accumulating in the food industry. C. glutamicum has been engineered to efficiently utilize both xylose and glucosamine as alternative carbon sources for growth and amino acid production (Gopinath et al. 2011; Meiswinkel et al. 2013a; Uhde et al. 2013; Matano et al. 2014).

In this study, the rational engineering of L-citrulline production by $C$. glutamicum is reported and the concept was extended to production of L-citrulline from the alternative carbon sources glucosamine, xylose, and starch.

\section{Materials and methods}

\section{Microorganisms and growth conditions}

Microorganisms and plasmids used in this study are listed in Table 1. E. coli DH5 $\alpha$ was used for gene cloning. C. glutamicum and E. coli strains were routinely grown in lysogeny broth (LB) $\left(10 \mathrm{~g} \mathrm{~L}^{-1}\right.$ tryptone, $5 \mathrm{~g} \mathrm{~L}^{-1}$ yeast extract, $10 \mathrm{~g} \mathrm{~L}^{-1}$ sodium chloride) in $500-\mathrm{mL}$ baffled flasks on a rotary shaker $(120 \mathrm{rpm})$ at $30^{\circ} \mathrm{C}$ or $37^{\circ} \mathrm{C}$. For growth experiments, CGXII minimal medium (Eggeling and Reyes 2005) was used for C. glutamicum. Growth was followed by measuring the optical density at $600 \mathrm{~nm}$ using a V-1200 Spectrophotometer (VWR, Radnor, PA, USA). An $\mathrm{OD}_{600}$ of 1 corresponds approximately to an estimated cell dry weight of $0.25 \mathrm{~g} / \mathrm{L}$.

When necessary, the growth medium was supplemented kanamycin $\left(25 \mu \mathrm{g} \mathrm{mL} L^{-1}\right)$, spectinomycin $\left(100 \mu \mathrm{g} \mathrm{mL}^{-1}\right)$, tetracycline $\left(10 \mu \mathrm{g} \mathrm{mL}^{-1}\right)$, isopropyl $\beta$-D-1-thiogalactopyranoside (IPTG) $(1 \mathrm{mM})$ and L-arginine $(750 \mu \mathrm{M})$. The growth behavior and L-citrulline production of recombinant C. glutamicum strains were analyzed in $500 \mathrm{ml}$ baffled flasks. Briefly, a $50 \mathrm{~mL}$ BHI $\left(37 \mathrm{~g} \mathrm{~L}^{-1}\right)$ seed culture was inoculated from an agar plate and grown overnight. The cells were harvested by centrifugation $(4,000 \times \mathrm{g}, 10 \mathrm{~min})$ and washed twice with CGXII minimal medium lacking the carbon source. Subsequently, $50 \mathrm{~mL}$ CGXII medium, containing a given concentration of carbon source and necessary supplements, was inoculated to an optical density of 1.0. Detailed information on the carbon source concentrations employed are given in the Results chapter.

\section{Molecular genetic techniques}

Standard methods such as restriction digestions, and ligation were carried out as described elsewhere (Sambrook and Russell 2012). Digested DNA was purified by using the QIAquick Gel Extraction Kit (Qiagen, Hilden, Germany).

\section{Table 1 Strains and plasmids used in this study}

\begin{tabular}{|c|c|c|}
\hline \multicolumn{3}{|l|}{ E. coli } \\
\hline $\mathrm{DH} 5 \mathrm{a}$ & 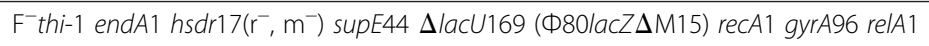 & (Hanahan 1983) \\
\hline \multicolumn{3}{|l|}{ C. glutamicum } \\
\hline MB001 & $\begin{array}{l}\text { ATCC } 13032 \text { with in-frame deletion of prophages CGP1 (cg1507-cg1524), } \\
\text { CGP2 (cg1746-cg1752), and CGP3 (cg1890-cg2071) }\end{array}$ & (Baumgart et al. 2013) \\
\hline CITO & MB001 with $\Delta \arg F, \Delta \arg G, \Delta \arg R$ & This study \\
\hline CIT1 & CITO carrying the pWWEx-argFB ${ }^{\mathrm{fbr}}$ vector & This study \\
\hline \multicolumn{3}{|l|}{ Plasmids } \\
\hline pEKEx3 & Spec $^{R}, P_{\text {tac }},\left.\right|_{a c l} ^{q}$ & (Stansen et al. 2005) \\
\hline pWWEx1 & $\mathrm{Kan}^{\mathrm{R}}, \mathrm{P}_{\mathrm{tac}}, \mathrm{lacl}^{\mathrm{q}}$ & (Peters-Wendisch et al. 2001) \\
\hline pEC-XT99A & Tet $^{\mathrm{R}}, \mathrm{P}_{\mathrm{trc}}, \mathrm{lacl}^{\mathrm{q}}$ & (Kirchner and Tauch 2003) \\
\hline pK19 $\Delta$ argFR & $\mathrm{Kan}^{\mathrm{R}}$, pk19mobsacB with the deletion construct of genes argFR & (Schneider et al. 2011) \\
\hline pK19 $\arg G$ & $\mathrm{Kan}^{\mathrm{R}}$, pk19mobsacB with the deletion construct of genes $\arg G$ & This study \\
\hline pEKEx3- $\arg B^{\mathrm{fbr}}$ & $\begin{array}{l}\text { Spec }^{\mathrm{R}} \text {, pEKEX3 carrying } \operatorname{argB} \text { from C. glutamicum ATCC } 13032 \text { with amino acid } \\
\text { exchanges A49VM54V }\end{array}$ & (Schneider et al. 2011) \\
\hline pWWEx1-argF & Kan $^{R}$, pWWEx1 carrying argF from C. glutamicum ATCC 13032 & This study \\
\hline pWWEx1-argFG & Kan $^{R}$, pWWEx1 carrying argF and argG from C. glutamicum ATCC 13032 & This study \\
\hline pWWEx1-argFB ${ }^{\mathrm{fbr}}$ & $\begin{array}{l}\text { Kan }^{\mathrm{R}}, \text { pWWEx1 carrying argF from C. glutamicum ATCC } 13032 \text { and } \arg B^{\mathrm{fbr}} \text { from } \\
\text { pEKEx3- } \arg B^{\text {fbr }}\end{array}$ & This study \\
\hline pEKEx3-nagB & Spec $^{R}$, pEKEx3 carrying nagB from C. glutamicum ATCC 13032 & (Uhde et al. 2013) \\
\hline pEKEx3-XyIAB & $\begin{array}{l}\text { Spec }^{R} \text {, pEKEx3 carrying XyIA from Xanthomonas campestris XCC } 1758 \text { and XyIB } \\
\text { from C. glutamicum ATCC } 13032\end{array}$ & (Meiswinkel et al. 2013a) \\
\hline PAMY & Tet $^{R}$, pEC-XT99A carrying amy from Streptomyces griseus IMRU 3570 & (Seibold et al. 2006) \\
\hline
\end{tabular}


E. coli cells were transformed by heat shock (Sambrook and Russell 2012) and C. glutamicum cells were transformed by electroporation (Eggeling and Reyes 2005). Isolation of genomic DNA was performed as previously described (Jensen and Wendisch 2013). Chromosomal changes in C. glutamicum were performed as described elsewhere (Eggeling and Reyes 2005).

\section{Construction of strains and plasmids}

The deletion of $\triangle a r g F R$ in MB001 was performed by using pK19mobsacB $\triangle \operatorname{argFR}$. Afterwards argG was deleted by using pK19mobsacB $\Delta a r g G$ to obtain CIT0. pK19mobsacB $\triangle \arg G$ contains the up- and downstream regions of $\operatorname{argG}$ in the $\triangle a r g F R$ strain. The plasmid was constructed by amplifying the upstream region with argG_up_f (CTTgaattcAGAAGCTGCGCCGCATG) and argG_up_r (agagacgacctaagccagtctAACGATGCGGTTAGTCATGAGG) and the downstream region with argG_down_f (agactggct taggtcgtctctGCTAACAAGCGCGATCGC) and argG_down_r (CCTctgcagAACGACCAGCGCGCAGA). The two fragments were combined by crossover PCR using argG_up_f and argG_down_r and finally cloned into pK19mobsacB with PstI and EcoRI.

pVWEx1-argF was constructed by amplifying $\arg F$ with primers argF_f (CTTgtcgacAAGGAGATATAGATATGAC TTCACAACCACAGGTTCG) and argF_r (CCTggatccTT ACCTCGGCTGGTTGGC). The PCR product was treated with SalI and BamHI and ligated with similarly treated pVWEx1. pVWEx1-argFG was constructed by amplifying argG with primers argG_f (GGGgtcgacGAAAGG AGGCCCTTCAGATGACTAACCGCATCGTTCTTG) and argG_r (GGGgtcgacTTAGTTGTTGCCAGCTTCG CGA). The PCR product was treated with SalI and ligated

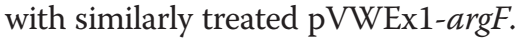

The plasmid vector pEKEx-argB ${ }^{\mathrm{fbr}} \quad\left(\arg B_{\mathrm{A} 49 \mathrm{VM} 54 \mathrm{~V}}\right.$ (Schneider et al. 2011)) was digested with BamHI and KpnI and the DNA fragment with a size of $0.9 \mathrm{~kb}$ harboring the $\arg B^{\mathrm{fbr}}$ gene was cloned into the BamHI/KpnI

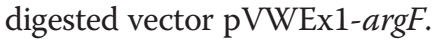

\section{Determination of amino acid and carbohydrate concentrations}

For the quantification of extracellular amino acids and carbohydrates, a high-performance liquid chromatography system was used (1200 series, Agilent Technologies Deutschland GmbH, Böblingen, Germany). Samples were withdrawn from the cultures, centrifuged $(13,000 \times \mathrm{g}$, $10 \mathrm{~min}$ ), and the supernatant used for analysis.

Glucose and xylose were analyzed on a normal phase column (organic acid resin $300 \times 8 \mathrm{~mm}, 10 \mu \mathrm{m}$ particle size, 25 A pore diameter; Chromatographie Service $\mathrm{GmbH}$, Langerwehe, Germany) using $5 \mathrm{mM}$ sulfuric acid as the

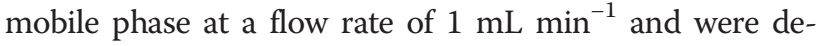
tected with a refractive index detector (RID G1362A, 1200 series, Agilent Technologies). Amino acids were automatically modified by precolumn derivatisation with orthophthalaldehyde and separated as described previously (Georgi et al. 2005). L-ornithine was quantified using a pre-column (LiChrospher $100 \mathrm{RP} 18 \mathrm{EC}-5 \mu(40 \times 4 \mathrm{~mm})$, CS-Chromatographie Service $\mathrm{GmbH}$, Langerwehe, Germany) and a reversed phase column (LiChrospher 100 RP18 EC-5 $\mu(125 \times 4 \mathrm{~mm})$, CS Chromatographie) as a main coulumn and detected with a fluorescence detector at excitation at $230 \mathrm{~nm}$ and $450 \mathrm{~nm}$ emission (FLD G1321A, 1200 series, Agilent Technologies). For the determination of L-citrulline, a reverse-phase (RP) LiChrospher 100 RP8 EC-5 $\mu$ precolumn $(40 \times 4.6 \mathrm{~mm})$ and a RP8 EC-5 $\mu$ $(125 \times 4.6 \mathrm{~mm})$ main column (CS Chromatographie, Langerwehe, Germany) were used. $100 \mu \mathrm{M}$ L-Asparagine was used as an internal standard. The mobile phases used were in case of RP8 A: $0.25 \% \mathrm{Na}$-acetate $\mathrm{pH} 6$, B: methanol. The gradient used was: $0 \mathrm{~min} 30 \% \mathrm{~B}, 1 \mathrm{~min} 30 \% \mathrm{~B}$, $6 \mathrm{~min}, 70 \%$ B, $11 \min 90 \%$ B, $14 \min 70 \%$ B, 16 min 30\% B. In case of RP18, the mobile phases used were A:0.1 M Na-acetate $\mathrm{pH} 7.2$, B: methanol. The gradient used was: $0 \min 20 \%$ B, $0.5 \min 38 \%$ B, 2.5 min $46 \%$ B, 3.7 min $65 \%$ B, $5.5 \min 70 \%$ B, 6 min $75 \%$ B, $6.2 \mathrm{~min} 85 \%$ B, $6.7 \mathrm{~min}$ $20 \%$ B.

\section{Results}

Engineering a prophage-free C. glutamicum strain for L-citrulline production

C. glutamicum has recently been cured of prophage sequences to yield MB001 (Baumgart et al. 2013). This strain was used as the parental strain because it can be transformed easily and plasmid-based gene overexpression is more efficient (Baumgart et al. 2013). As C. glutamicum ATCC 13032, this strain does not accumulate L-citrulline, an intermediate of L-arginine biosynthesis (Figure 1). The deletion of three genes of the L-arginine operon (L-ornithine carbamoyltransferase (EC 2.1.3.3) $\operatorname{argF}$, argininosuccinate synthetase (EC 6.3.4.5) $\arg G$, and L-arginine biosynthesis operon repressor gene $\operatorname{argR}$ ) in C. glutamicum MB001 yielded the L-arginine auxotrophic strain CIT0 (Table 1). When supplemented with $0.75 \mathrm{mM}$ L-arginine, C. glutamicum CIT0 accumulated $25.2 \pm 2.6 \mathrm{mM}$ L-ornithine from $2 \%$ glucose (Table 2). The deletion of $\operatorname{argF}$ and $\arg G$ could be complemented by plasmid-borne expression of these genes since the complemented strain CIT0(pVWEx1-argFG) grew without L-arginine supplement while the empty vector carrying control CIT0(pVWEx1) did not (data not shown). Comparable growth rates and biomass concentrations were observed.

To enable L-citrulline accumulation, two plasmids were constructed and used to transform C. glutamicum CIT0. While pVWEx1-argF only carries $\arg F$ encoding L-ornithine carbamoyltransferase, pVWEx1-argFB ${ }^{\mathrm{fbr}}$ in addition carries 
Table 2 Growth on different carbon sources

\begin{tabular}{llll}
\hline C. glutamicum strain & Carbon source concentration & Maximum OD $_{\mathbf{6 0 0}}$ & Growth rate $^{\left(\mathbf{h}^{\mathbf{1}} \text { ) }\right.}$ \\
\hline CIT1(pEKEX3-XylAB) & Xylose: $15 \mathrm{~g} / \mathrm{L}$ & $6 \pm 1$ & $0.03 \pm 0.01$ \\
CIT1(pEKEX3-nagB) & Glucosamine: $10 \mathrm{~g} / \mathrm{L}$ & $3 \pm 1$ & $0.02 \pm 0.01$ \\
CIT1(pAMY) & Soluble starch: $10 \mathrm{~g} / \mathrm{L}$ Glucose: $2.5 \mathrm{~g} / \mathrm{L}$ & $9 \pm 1$ & $0.21 \pm 0.01$ \\
CIT1(pEC-XT99A) & Soluble starch: $10 \mathrm{~g} / \mathrm{L}$ Glucose: $2.5 \mathrm{~g} / \mathrm{L}$ & $3 \pm 1$ & $0.10 \pm 0.01$ \\
\hline
\end{tabular}

Fermentations were performed in CGXII minimal medium containing the respective carbon source and were supplemented by $750 \mu \mathrm{M}$ L-arginine. 1 mM IPTG and $25 \mu \mathrm{g} / \mathrm{ml}$ kanamycin and spectinomycin were added. Values and error bars represent the mean and the standard error of triplicates.

$\arg B^{\mathrm{fbr}}$ encoding feedback-resistant N-acetyl L-glutamate kinase (NAGK, EC 2.7.2.8). When grown in minimal medium with $2 \%$ glucose and $0.75 \mathrm{mM}$ L-arginine C. glutamicum CIT0(pVWEx1-argF) grew to a higher OD than CIT0(pVWEx1) (Figure 2) and did not accumulate notable concentrations of L-citrulline. As opposed to CIT0 (pVWEx1), CIT0(pVWEx1-argF) did not produce Lornithine (Figure 3). By contrast, the combined overexpression of $\operatorname{argF}$ and $\arg B^{\mathrm{fbr}}$ entailed L-citrulline production and the respective strain was named CIT1. C. glutamicum CIT1 accumulated $44.1 \pm 0.5 \mathrm{mM}$ L-citrulline in minimal medium with $2 \%$ glucose (Figure 4 ).

When comparing the growth of C. glutamicum CIT0 (pVWEx1) to that of CIT0(pVWEx1-argF), similar growth rates $\left(0.37 \pm 0.01 h^{-1}\right.$ and $0.35 \pm 0.04 h^{-1}$, respectively) were obtained, whereas L-citrulline formation by CIT0 (pVWEx1-argFB ${ }^{\mathrm{fbr}}$ ) was accompanied by a reduced growth rate $\left(0.15 \pm 0.01 \mathrm{~h}^{-1}\right)$ (Figure 1$)$. Moreover, the final $\mathrm{OD}_{600}$ of CIT0(pVWEx1-argFB ${ }^{\mathrm{fbr}}$ ) was $20 \pm 1$ as compared to an $\mathrm{OD}_{600}$ of $26 \pm 1$ of CIT0(pVWEx1). By contrast, C. glutamicum CIT0(pVWEx1-argF) grew

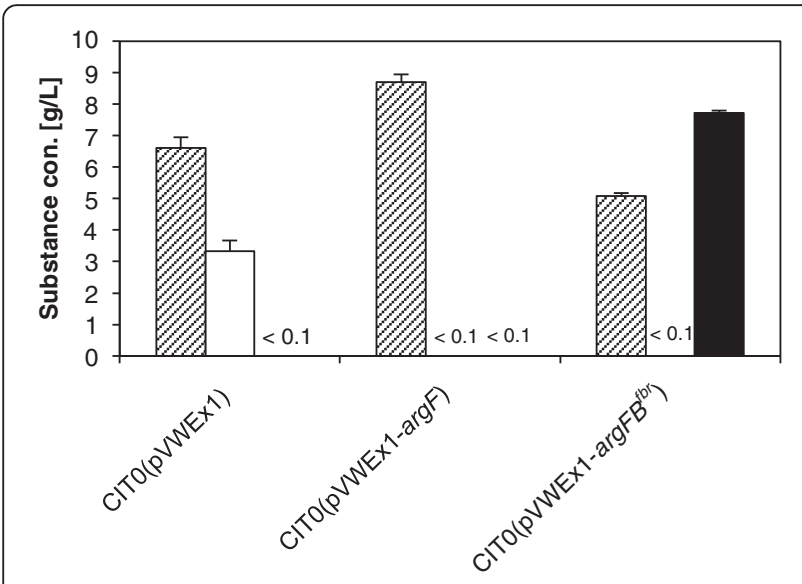

Figure 3 Biomass formation and production of ornithine and citrulline on glucose by various C. glutamicum strains: cell dry weight (hatched bars), L-ornithine concentration (open bars) and L-citrulline concentration (filled bars). The cultivation was performed in CGXII minimal medium containing $20 \mathrm{~g} / \mathrm{L}$ glucose, $1 \mathrm{mM}$ IPTG, $750 \mu \mathrm{M} \mathrm{L}$-arginine and $25 \mu \mathrm{g} / \mathrm{L}$ kanamycin. The amino acid concentrations in the supernatant were determined after the consumption of glucose. Values and error bars represent the mean and the standard error of triplicates. to a higher biomass concentration with a final $\mathrm{OD}_{600}$ of $35 \pm 1$. As shown in Figure 3, the lower growth rates of CIT0(pVWEx1) and CIT0(pVWEx1-argFB ${ }^{\mathrm{fbr}}$ ) correlated inversely with the formation of the respective amino acids L-ornithine and L-citrulline, whereas C. glutamicum CIT0(pVWEx1-argF) reaches a higher final biomass and neither produces L-ornithine nor L-citrulline.

Production of L-citrulline from alternative carbon sources Due to the high demand of biotechnological processes of using complex sugar substrates derived from raw materials and industrial wastes, the L-citrulline producer strain CIT1 was enabled to utilize the alternative carbon sources starch (as an example of a high molecular weight carbohydrate), xylose, and glucosamine (as an example of a carbohydrates, derived from forestry and food industrial wastes).

To enable $C$. glutamicum CIT1 to consume starch, the gene amyA from Streptomyces griseus was overexpressed. The combined overexpression of $x y l A$ from Xanthomonas campestris and endogenous $x y l B$ allowed the utilization of xylose by $C$. glutamicum CIT1. The endogenous nagB was overpressed ectopically to facilitate the consumption of glucosamine. The resulting strains were tested for growth and L-citrulline production.

When cultured in CGXII medium supplemented $0.75 \mathrm{mM} \mathrm{L}$-arginine all strains engineered for alternative carbon source consumption grew with their respective substrate (Table 1 ). The empty vector carrying strain CIT1(pEKEx3) neither grew in xylose or glucosamine minimal medium nor consumed these substrates. By contrast, the recombinant strain CIT1(pEKEx3-xylAB) grew in xylose minimal medium with a growth rate of $0.03 \pm$ $0.01 \mathrm{~h}^{-1}$ and reached a final $\mathrm{OD}_{600}$ of $6 \pm 1$. In glucosamine minimal medium, C. glutamicum CIT1(pEKEx3-nagB) grew to a final $\mathrm{OD}_{600}$ of $3 \pm 1$ with a growth rate of $0.02 \pm 0.01 \mathrm{~h}^{-1}$. In minimal medium containing $1 \%$ starch and $0.25 \%$ glucose as carbon sources, the empty vector harbouring strain CIT1(pEC-XT99A) formed roughly one third of the biomass as compared to C. glutamicum CIT1(pAmy). Growth of CIT1(pEC-XT99A) was slower (growth rate of $0.10 \pm 0.01 \mathrm{~h}^{-1}$ ) than that of CIT1(pAmy) (growth rate of $0.21 \pm 0.01 \mathrm{~h}^{-1}$ ). While strain CIT1(pEC- 

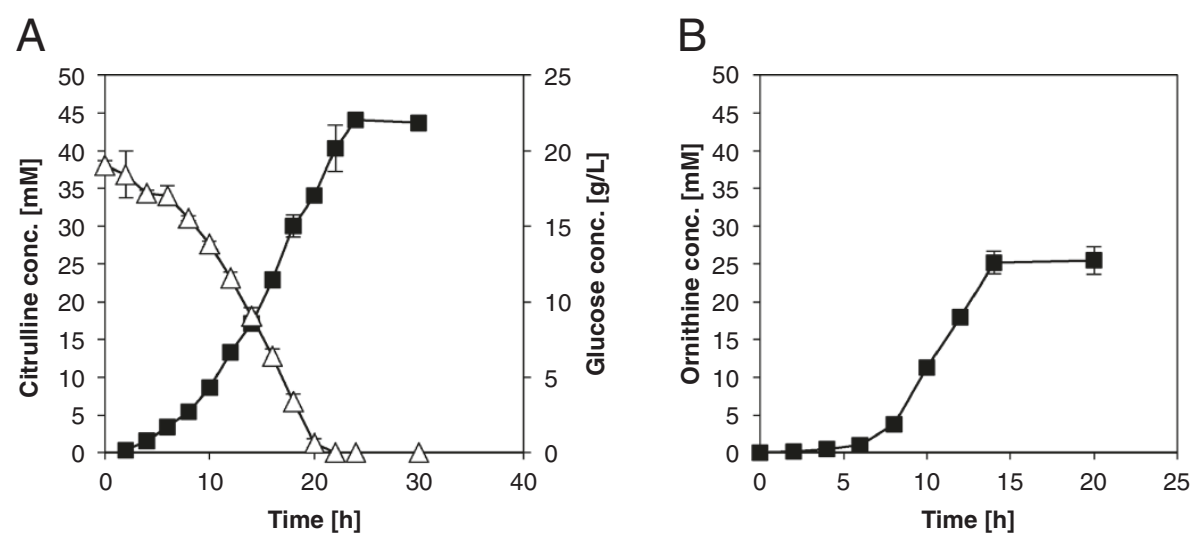

Figure 4 Amino acid production by various C. glutamicum strains. L-ornithine production by C. glutamicum CITO(pWWEx1) (filled squares) (A) and L-citrulline accumulation (filled squares) and glucose consumption (open triangles) by strain CITO(pWWEx1-argFB $B^{\text {fbr }}$ ) (B). The experiments were performed in CGXII minimal medium with $20 \mathrm{~g} / \mathrm{L}$ glucose, $1 \mathrm{mM} \mathrm{IPTG}, 25 \mu \mathrm{g} / \mathrm{L}$ kanamycin and supplemented with $750 \mu \mathrm{M} \mathrm{L}$-arginine. Values and error bars represent the mean and the standard error of triplicates.

XT99A) only utilized glucose, but not starch, CIT1(pAmy) was able to consume both, glucose and starch.

The strains engineered for utilization of xylose and glucosamine, respectively, also produced L-citrulline from these carbon sources (Figure 5). C. glutamicum CIT1(pEKEx3$n a g B)$ accumulated $2.6 \pm 0.3 \mathrm{mM}$ L-citrulline which corresponds to a yield of $0.045 \pm 0.002 \mathrm{~g} / \mathrm{g}$ since glucosamine was utilized completely. Similarly, after complete utilization of xylose by C. glutamicum CIT1(pEKEx3-xylAB) $6.4 \pm$ $0.1 \mathrm{mM}$ L-citrulline accumulated corresponding to a yield of $0.075 \pm 0.001 \mathrm{~g}$ per $\mathrm{g}$ xylose.
As the determination of the starch concentration by HPLC was not possible, residual starch content was assayed by the use of Lugols solution. However, as it is known that overexpression of amyA in C. glutamicum results in high molecular mass degradation products of starch, which remain in the medium and are not detectable by Lugols solution (Seibold et al. 2006), the L-citrulline concentration was measured until no change in $\mathrm{OD}_{600}$, starch content and L-citrulline concentration was observed. The starch utilizing strain CIT1(pAmy) was able to produce $11.9 \pm 0.5 \mathrm{mM}$ L-citrulline which corresponds to a yield of $0.167 \mathrm{~g} / \mathrm{g}$.

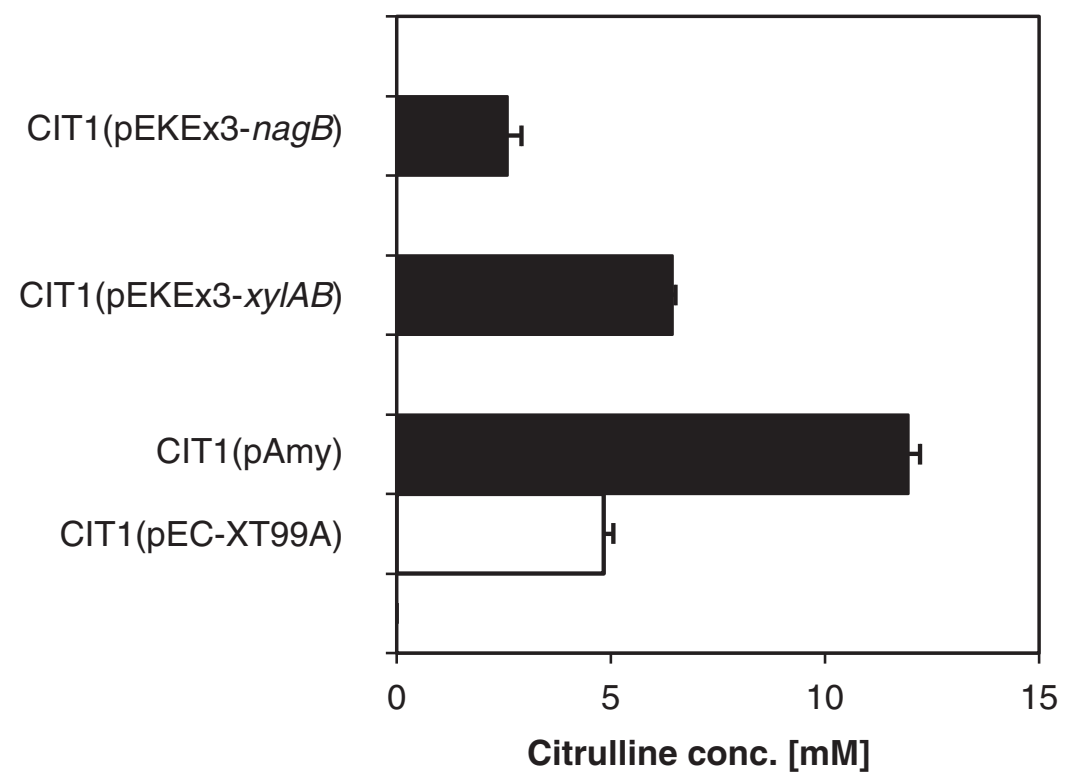

Figure 5 L-citrulline concentration in the engineered strains after the consumption of the respective carbon source. CIT1(pEC-XT99A), CIT1(pAmy) with $10 \mathrm{~g} / \mathrm{L}$ soluble starch, 2,5 g/L glucose after 31 h. CIT1(pEKEx2-xy/AB) with $15 \mathrm{~g} / \mathrm{L}$ xylose after xylose consumption. CIT1 (pEKEx3-nagB) with $10 \mathrm{~g} / \mathrm{L}$ glucosamine after glucosamine consumption. Values and error bars represent the mean and the standard error of triplicates. 


\section{Discussion}

C. glutamicum was engineered to accumulate L-citrulline as major product, both from glucose as well as from the alternative carbon sources starch, glucosamine and xylose.

Feedback insensitive $\mathrm{N}$-acetyl L-glutamate kinase (encoded by $\operatorname{argB}^{\mathrm{fbr}}$; (A49VM54V)) was required for production of L-citrulline since CIT0(pVWEx1-argF) did not produce L-citrulline, while CIT0(pVWEx1-argFB $\left.{ }^{\mathrm{fbr}}\right)$ produced L-citrulline. It is unlikely that addition of L-arginine to CIT0(pVWEx1-argF) inhibited generation of L-ornithine, a precursor of L-citrulline, because strain CIT0(pVWEx1) produced L-ornithine when supplemented with L-arginine. However, it is possible that intracellular L-citrulline affects arginine biosynthesis. As overexpression of $\arg B^{\mathrm{fbr}}$ entailed L-citrulline formation, we assume that L-citrulline inhibits the NAGK of C. glutamicum, but this has not yet been described. As expected due to its structural similarity to L-arginine, L-citrulline inhibits NAGK of other microorganisms (Farago and Denes 1967; Haas and Leisinger 1975). In Chlamydomonas reinhardtii, NAGK is inhibited by several L-arginine structure analogs, including L-citrulline, however, inhibition was less pronounced than L-arginine inhibition (Farago and Denes 1967). NAGK from Pseudomonas aeruginosa lost two thirds of its activity in the presence of $2.5 \mathrm{mM}$ L-citrulline which was claimed to be too weak under physiologic conditions (Haas and Leisinger 1975). However, it is conceivable that inhibition of NAGK by L-citrulline may play a role in recombinant C. glutamicum strains engineered for L-citrulline production, thus, possibly explaining the finding that L-citrulline production required overexpression $\arg B^{\mathrm{fbr}}$ encoding NAGK feedback resistant to L-arginine. Commensurate with this notion, simultaneous production of L-arginine and L-citrulline resulted from $\arg B^{\mathrm{fbr}}$ overexpression in a $\triangle a r g R$ background (Ikeda et al. 2009). In this $\arg B^{\mathrm{fbr}}$ overexpressing strain, the ratio of L-citrulline to L-arginine was higher than by classically obtained strains, which solely contain native $\operatorname{argB}$ (Ikeda et al. 2009). Currently, it remains to be studied if L-citrulline inhibits NAGK from C. glutamicum and if (some) variants feed-back resistant to L-arginine are also desensitized to L-citrulline.

Notably, about two fold more L-citrulline (about $7.7 \mathrm{~g} / \mathrm{L}$ ) was produced by strain CIT1 than L-ornithine was produced (about $3.3 \mathrm{~g} / \mathrm{L}$ ) by the isogenic strain CIT0 (pVWEx1). Both, overexpression of $\arg F$ and $\arg B^{\mathrm{fbr}}$ may have contributed to this effect. It is more likely that $\arg B^{\mathrm{fbr}}$ is responsible as L-arginine supplementation may have limited flux in the arginine biosynthesis pathway of strain CIT0(pVWEx1) especially in the beginning of the cultivation. In C. glutamicum CIT1, only feedbackresistant NAGK is present and additionally a gene dosage effect due ectopic overexpression of $\arg B^{\mathrm{fbr}}$ might have contributed to increase L-citrulline production.
Glucose, glucosamine, xylose, and starch were shown to be suitable substrates for the production of L-citrulline. Strain construction was based on previously established engineering strategies (Seibold et al. 2006; Uhde et al. 2013; Meiswinkel et al. 2013a; Gopinath et al. 2011). The achieved L-citrulline concentrations on these substrates were lower than with glucose as carbon source. However, L-citrulline production from xylose $(6.44 \pm 0.12 \mathrm{mM})$ by CIT1(pEKEx3- $x y l A B$ ) was lower, but in a similar range as production of L-ornithine $(19.6 \pm 1.9 \mathrm{mM})$ and putrescine $(15.1 \pm 1.2 \mathrm{mM})$, respectively, from the same xylose concentration by the respective recombinant C. glutamicum strains (Meiswinkel et al. 2013a). Similarly, product yields with glucosamine as carbon source were lower for L-citrulline $(0.067 \mathrm{~g} / \mathrm{g})$ than for putrescine $(0.112 \mathrm{~g} / \mathrm{g})$ (Uhde et al. 2013). Unexpectedly and hitherto not understood, the growth rate $\left(0.02 \pm 0.01 \mathrm{~h}^{-1}\right)$ and, thus, productivity by CIT1(pEKEx3-nagB) were very low. By contrast, a putrescine producing strain carrying pEKEx3-nagB showed only a slightly decreased growth rate (Uhde et al. 2013).

C. glutamicum strains carrying pAMY co-utilized starch with glucose (Seibold et al. 2006). Substrate co-utilization is observed with C. glutamicum WT as well as recombinant strains for almost all mixtures of carbon sources (Blombach and Seibold 2010). A L-lysine producing strain carrying pAMY showed increased biomass formation by addition of $10 \mathrm{~g} / \mathrm{L}$ starch to $10 \mathrm{~g} / \mathrm{L}$ glucose, whereas L-lysine production increased only upon addition of higher starch concentrations (Seibold et al. 2006).

In this study, the additional presence of starch increased the growth rate of CIT1 (from 0.15 to $0.21 \mathrm{~h}^{-1}$ ) as well as L-citrulline production. Production of L-citrulline by CIT1(pAMY) from a starch glucose mixture was higher $(11.95 \pm 0.48 \mathrm{mM})$ than that by the empty vector carrying control strain $(4.83 \pm 0.4 \mathrm{mM})$ demonstrating that starch contributed to production of L-citrulline. It has to be noted that starch cannot be utilized completely by C. glutamicum strains overexpressing the $\alpha$-amylase gene amy $A$ because high-molecular-weight carbohydrates are generated from starch and remain unutilized in the medium (Seibold et al. 2006).

Taken together, production of L-citrulline as major product from glucose, starch, glucosamine, and xylose by recombinant C. glutamicum strains was achieved.

\section{Competing interests}

The authors declare that they have no competing interests.

\section{Authors' contributions}

DE designed experiments, performed experiments, analysed results and drafted the manuscript. JVKJ designed experiments, performed experiments and analysed results. VFW coordinated the study, designed experiments, analysed results and wrote the manuscript. All authors read and approved the final manuscript.

\section{Acknowledgment}

This work was partially supported by the Bundesministerium für Bildung und Forschung (BMBF, grant. no. 0316017) and by the program ZIM (grant. no. 
KF2969003SB2). We acknowledge support for the Article Processing Charge by the Deutsche Forschungsgemeinschaft and the Open Access Publication Fund of Bielefeld University.

Received: 13 October 2014 Accepted: 20 November 2014 Published online: 10 December 2014

\section{References}

An SJ, Yim SS, Jeong KJ (2013) Development of a secretion system for the production of heterologous proteins in Corynebacterium glutamicum using the Porin B signal peptide. Protein Expr Purif 89(2):251-257, doi:10.1016/j. pep.2013.04.003

Arndt A, Eikmanns BJ (2008) Regulation of carbon metabolism in Corynebacterium glutamicum. In: Burkovski A (ed) Corynebacteria: genomics and molecular biology. Caister Academic Press, Wymondham, UK, pp 155-182

Baumgart M, Unthan S, Ruckert C, Sivalingam J, Grunberger A, Kalinowski J, Bott M, Noack S, Frunzke J (2013) Construction of a prophage-free variant of Corynebacterium glutamicum ATCC 13032 for use as a platform strain for basic research and industrial biotechnology. Appl Environ Microbiol 79(19):6006-6015, doi:10.1128/AEM. 01634-13

Blombach B, Seibold GM (2010) Carbohydrate metabolism in Corynebacterium glutamicum and applications for the metabolic engineering of L-lysine production strains. Appl Microbiol Biotechnol 86(5):1313-1322, doi:10.1007/ s00253-010-2537-z

Blombach B, Hans S, Bathe B, Eikmanns BJ (2009) Acetohydroxyacid synthase, a novel target for improvement of L-lysine production by Corynebacterium glutamicum. Appl Environ Microbiol 75(2):419-427, doi:10.1128/AEM. 01844-08

Curis E, Nicolis I, Moinard C, Osowska S, Zerrouk N, Benazeth S, Cynober L (2005) Almost all about citrulline in mammals. Amino Acids 29(3):177-205, doi:10.1007/s00726-005-0235-4

Delaunay S, Gourdon P, Lapujade P, Mailly E, Oriol E, Engasser JM, Lindley NL, Goergen $J$ (1999) An improved temperature triggered process for glutamate production with Corynebacterium glutamicum. Enz Microb Biotechnol 25:762-768

Eggeling L, Reyes O (2005) Experiments. In: Eggeling L, Bott M (eds) Handbook of Corynebacterium glutamicum. CRC Press, Boca Raton, USA, pp 3535-3566

Farago A, Denes G (1967) Mechanism of arginine biosynthesis in Chlamydomonas reinhardti. II. Purification and properties of $\mathrm{N}$-acetylglutamate 5phosphotransferase, the allosteric enzyme of the pathway. Biochim Biophys Acta 136(1):6-18

Fish WW (2012) Process for the production of L-citrulline from watermelon flesh and rind. US 8173837 B1

Frohwitter J, Heider SA, Peters-Wendisch P, Beekwilder J, Wendisch VF (2014) Production of the sesquiterpene (+)-valencene by metabolically engineered Corynebacterium glutamicum. J Biotechnol. doi:10.1016/j.jbiotec.2014.05.032

Georgi T, Rittmann D, Wendisch VF (2005) Lysine and glutamate production by Corynebacterium glutamicum on glucose, fructose and sucrose: roles of malic enzyme and fructose-1,6-bisphosphatase. Metab Eng 7(4):291-301

Gopinath V, Meiswinkel TM, Wendisch VF, Nampoothiri KM (2011) Amino acid production from rice straw and wheat bran hydrolysates by recombinant pentose-utilizing Corynebacterium glutamicum. Appl Microbiol Biotechnol. doi:10.1007/s00253-011-3478-x

Haas D, Leisinger T (1975) N-acetylglutamate 5-phosphotransferase of Pseudomonas aeruginosa. Catalytic and regulatory properties. Eur J Biochem 52(2):377-393

Hanahan D (1983) Studies on transformation of Escherichia coli with plasmids. J Mol Biol 166(4):557-580

Heider SA, Peters-Wendisch P, Netzer R, Stafnes M, Brautaset T, Wendisch VF (2014a) Production and glucosylation of C50 and C 40 carotenoids by metabolically engineered Corynebacterium glutamicum. Appl Microbiol Biotechnol 98(3):1223-1235, doi:10.1007/s00253-013-5359-y

Heider SA, Peters-Wendisch P, Wendisch VF, Beekwilder J, Brautaset T (2014b) Metabolic engineering for the microbial production of carotenoids and related products with a focus on the rare $\mathrm{C} 50$ carotenoids. Appl Microbiol Biotechnol 98(10):4355-4368, doi:10.1007/s00253-014-5693-8

Ikeda M, Mitsuhashi S, Tanaka K, Hayashi M (2009) Reengineering of a Corynebacterium glutamicum L-arginine and L-citrulline producer. Appl Environ Microbiol 75(6):1635-1641, doi:10.1128/AEM. 02027-08

Jensen JV, Wendisch VF (2013) Ornithine cyclodeaminase-based proline production by Corynebacterium glutamicum. Microb Cell Factories 12:63, doi:10.1186/1475-2859-12-63
Jolkver E, Emer D, Ballan S, Kramer R, Eikmanns BJ, Marin K (2009) Identification and characterization of a bacterial transport system for the uptake of pyruvate, propionate, and acetate in Corynebacterium glutamicum. J Bacteriol 191(3):940-948, doi:10.1128/JB.01155-08

Kakimoto T, Shibatani T, Nishimura N, Chibata I (1971) Enzymatic production of L-citrulline by Pseudomonas putida. Appl Microbiol 22(6):992-999

Kikuchi Y, Itaya H, Date M, Matsui K, Wu LF (2009) TatABC overexpression improves Corynebacterium glutamicum Tat-dependent protein secretion. Appl Environ Microbiol 75(3):603-607, doi:10.1128/AEM. 01874-08

Kim J, Hirasawa T, Sato Y, Nagahisa K, Furusawa C, Shimizu H (2009) Effect of odhA overexpression and odhA antisense RNA expression on Tween-40triggered glutamate production by Corynebacterium glutamicum. Appl Microbio Biotechnol 81(6):1097-1106, doi:10.1007/s00253-008-1743-4

Kim J, Fukuda H, Hirasawa T, Nagahisa K, Nagai K, Wachi M, Shimizu H (2010) Requirement of de novo synthesis of the Odhl protein in penicillin-induced glutamate production by Corynebacterium glutamicum. Appl Microbiol Biotechnol 86(3):911-920, doi:10.1007/s00253-009-2360-6

Kirchner O, Tauch A (2003) Tools for genetic engineering in the amino acid-producing bacterium Corynebacterium glutamicum. J Biotechnol 104(1-3):287-299

Matano C, Uhde A, Youn JW, Maeda T, Clermont L, Marin K, Kramer R, Wendisch VF, Seibold GM (2014) Engineering of Corynebacterium glutamicum for growth and L-lysine and lycopene production from N-acetyl-glucosamine. Appl Microbiol Biotechnol 98(12):5633-5643, doi:10.1007/s00253-014-5676-9

Meiswinkel TM, Gopinath V, Lindner SN, Nampoothiri KM, Wendisch VF (2013a) Accelerated pentose utilization by Corynebacterium glutamicum for accelerated production of lysine, glutamate, ornithine and putrescine. Microb Biotechnol 6(2):131-140, doi:10.1111/1751-7915.12001

Meiswinkel TM, Rittmann D, Lindner SN, Wendisch VF (2013b) Crude glycerol-based production of amino acids and putrescine by Corynebacterium glutamicum. Bioresour Technol 145:254-258, doi:10.1016/j.biortech.2013.02.053

Okumura S, Shibuya M, Shimpachi K, Teruo S, Noboru K (1966) Method of producing citrulline by bacterial fermentation. US $3282794 \mathrm{~A}$

Peters-Wendisch PG, Kreutzer C, Kalinowski J, Patek M, Sahm H, Eikmanns BJ (1998) Pyruvate carboxylase from Corynebacterium glutamicum: characterization, expression and inactivation of the pyc gene. Microbiology 144(Pt 4):915-927

Peters-Wendisch PG, Schiel B, Wendisch VF, Katsoulidis E, Mockel B, Sahm H, Eikmanns BJ (2001) Pyruvate carboxylase is a major bottleneck for glutamate and lysine production by Corynebacterium glutamicum. J Mol Microbiol Biotechnol 3(2):295-300

Radmacher E, Stansen KC, Besra GS, Alderwick LJ, Maughan WN, Hollweg G, Sahm H, Wendisch VF, Eggeling L (2005) Ethambutol, a cell wall inhibitor of Mycobacterium tuberculosis, elicits L-glutamate efflux of Corynebacterium glutamicum. Microbiology 151(Pt 5):1359-1368

Rimando AM, Perkins-Veazie PM (2005) Determination of citrulline in watermelon rind. J Chromatogr A 1078(1-2):196-200

Rittmann D, Lindner SN, Wendisch VF (2008) Engineering of a glycerol utilization pathway for amino acid production by Corynebacterium glutamicum Appl Environ Microbiol 74(20):6216-6222, doi:10.1128/AEM. 00963-08

Sakanyan V, Petrosyan P, Lecocq M, Boyen A, Legrain C, Demarez M, Hallet JN, Glansdorff N (1996) Genes and enzymes of the acetyl cycle of arginine biosynthesis in Corynebacterium glutamicum: enzyme evolution in the early steps of the arginine pathway. Microbiology 142(Pt 1):99-108

Sambrook J, Russell D (2012) Molecular cloning. A laboratory manual, 4th edn. Cold Spring Harbor Laboratoy Press, Cold Spring Harbor, NY

Sasaki M, Teramoto H, Inui M, Yukawa H (2011) Identification of mannose uptake and catabolism genes in Corynebacterium glutamicum and genetic engineering for simultaneous utilization of mannose and glucose. Appl Microbiol Biotechnol 89(6):1905-1916, doi:10.1007/s00253-010-3002-8

Sato H, Orishimo K, Shirai T, Hirasawa T, Nagahisa K, Shimizu H, Wachi M (2008) Distinct roles of two anaplerotic pathways in glutamate production induced by biotin limitation in Corynebacterium glutamicum. J Biosci Bioeng 106(1):51-58

Schneider J, Wendisch VF (2010) Putrescine production by engineered Corynebacterium glutamicum. Appl Microbiol Biotechnol 88(4):859-868, doi:10.1007/s00253-010-2778-x

Schneider J, Niermann K, Wendisch VF (2011) Production of the amino acids L-glutamate, L-lysine, L-ornithine and $\mathrm{L}$-arginine from arabinose by recombinant Corynebacterium glutamicum. J Biotechnol 154(2-3):191-198, doi:10.1016/j. jbiotec.2010.07.009 
Schneider J, Eberhardt D, Wendisch VF (2012) Improving putrescine production by Corynebacterium glutamicum by fine-tuning ornithine transcarbamoylase activity using a plasmid addiction system. Appl Microbiol Biotechnol 95(1):169-178, doi:10.1007/s00253-012-3956-9

Seibold G, Auchter M, Berens S, Kalinowski J, Eikmanns BJ (2006) Utilization of soluble starch by a recombinant Corynebacterium glutamicum strain: growth and lysine production. J Biotechnol 124(2):381-391

Stansen C, Uy D, Delaunay S, Eggeling L, Goergen JL, Wendisch VF (2005)

Characterization of a Corynebacterium glutamicum lactate utilization operon induced during temperature-triggered glutamate production. Appl Environ Microbiol 71(10):5920-5928

Tateno T, Fukuda H, Kondo A (2007) Production of L-Lysine from starch by Corynebacterium glutamicum displaying alpha-amylase on its cell surface. Appl Microbiol Biotechnol 74(6):1213-1220, doi:10.1007/s00253-006-0766-y

Teramoto H, Watanabe K, Suzuki N, Inui M, Yukawa H (2011) High yield secretion of heterologous proteins in Corynebacterium glutamicum using its own Tat-type signal sequence. Appl Microbiol Biotechnol 91(3):677-687, doi:10.1007/s00253-011-3281-8

Tsuge Y, Tateno T, Sasaki K, Hasunuma T, Tanaka T, Kondo A (2013) Direct production of organic acids from starch by cell surface-engineered Corynebacterium glutamicum in anaerobic conditions. AMB Express 3(1):72, doi:10.1186/2191-0855-3-72

Uhde A, Youn JW, Maeda T, Clermont L, Matano C, Kramer R, Wendisch VF, Seibold GM, Marin K (2013) Glucosamine as carbon source for amino acid-producing Corynebacterium glutamicum. Appl Microbiol Biotechnol 97(4):1679-1687, doi:10.1007/s00253-012-4313-8

Wada M (1930) Über Citrullin, eine neue Aminosäure im Presssaft der Wassermelone, Citrullus vulgaris Schrad. Biochem Z 224:420-429

Wendisch VF (2014) Microbial production of amino acids and derived chemicals: synthetic biology approaches to strain development. Curr Opin Biotechnol 30C:51-58, doi:10.1016/j.copbio.2014.05.004

Wendisch VF, Eberhardt D, Herbst M, Jensen JVK (2014) Amino acids and nucleotides. In: Bicas J (ed) Biotechnological production of natural ingredients for food industry. Bentham eBooks.

Yamamoto K, Sato T, Tosa T, Chibata I (1974) Continuous production of L-citrulline by immobilized Pseudomonas putida cells. Biotechnol Bioeng 16(12):1589-1599, doi:10.1002/bit.260161203

doi:10.1186/s13568-014-0085-0

Cite this article as: Eberhardt et al:: L-citrulline production by metabolically engineered Corynebacterium glutamicum from glucose and alternative carbon sources. AMB Express 2014 4:85.

\section{Submit your manuscript to a SpringerOpen ${ }^{\circ}$ journal and benefit from:}

- Convenient online submission

- Rigorous peer review

- Immediate publication on acceptance

- Open access: articles freely available online

- High visibility within the field

- Retaining the copyright to your article

Submit your next manuscript at $\gg$ springeropen.com 